\title{
Domestic violence among female psychiatric patients: cross-sectional survey
}

\author{
John F. Morgan, ${ }^{1,2}$ Gabriella Zolese, ${ }^{3}$ Jane McNulty, ${ }^{4}$ Sharon Gebhardt ${ }^{5}$
}

The Psychiatrist (2010), 34, 461-464, doi: 10.1192/pb.bp.108.023986

${ }^{1}$ Leeds Partnerships NHS Foundation Trust, Seacroft Hospital; ${ }^{2}$ St George's University, London: ${ }^{3}$ South West London and St George's Mental Health NHS Trust; ${ }^{4}$ Sussex Partnership NHS Foundation Trust; ${ }^{5}$ Surrey and Borders Partnership NHS Foundation Trust Correspondence to John F. Morgan (john.morgan@leedspft.nhs.uk)

\begin{abstract}
Aims and method To establish prevalence of domestic violence among female psychiatric patients, including risk factors, health professional attention and acceptability of routine enquiry. Participants were 70 adult women in an inner-city community mental health team who received questionnaire and case-note review. Main outcome measures were: lifetime/point prevalence of domestic violence; attitudes to routine enquiry; past disclosure and recording in psychiatric records; clinical and demographic risk factors.
\end{abstract}

Results Lifetime prevalence was $60 \%$ for physical violence from partners, $27 \%$ during pregnancy and $40 \%$ receiving injuries. Point prevalence was not reported, as an insufficient number of participants were currently in a relationship. As many as $82 \%$ regarded routine enquiry as acceptable, but only $24 \%$ had ever been questioned. Logistic regression analysis showed prediction by presence of children, previous overdose, and experience of sexual abuse.

Clinical implications Domestic violence in female psychiatric patients is common but undetected. Enquiry should be routine, but would require staff training.

Declaration of interest None.
Domestic violence presents a heavy personal, societal and fiscal burden. The World Health Organization (WHO) published a multicountry study and reported high rates of violence against women, recommending detection and prevention as a priority, ${ }^{1}$ with reported lifetime prevalence of partner violence varying from 15 to $71 \%$. Studies in UK accident and emergency and primary care settings have found high prevalence of domestic violence with elevated risks of consequent mental disorders. ${ }^{2,3}$ Extrapolation would predict overrepresentation among female psychiatric patients, assuming causative association. ${ }^{4}$ Indeed, prevalence of domestic violence preceding psychiatric admission has been recorded as $63 \%$ in the USA. ${ }^{5}$ Women receiving psychiatric treatment are vulnerable to traumatic exploitation. ${ }^{6}$ However, to our knowledge there has only been one British study examining prevalence in women attending psychiatric services, and this addressed acute mental health assessments. ${ }^{7}$ We therefore aimed to measure prevalence of domestic violence among female psychiatric patients in an urban catchment area, including risk factors, health professional attention and acceptability of questioning, using a structured questionnaire adapted from that applied in a study in primary care in the UK. ${ }^{2}$

\section{Method}

All female patients (aged 18 and over) treated by a community mental health team covering a locality in the London borough of Wandsworth in a 12-month period (2003) were surveyed. We excluded women with intellectual disability, dementia and those unable to give consent. The study had approval from the local research ethics committee. Participants gave written informed consent to complete the questionnaire and for researchers to review their case notes.

\section{Case definition}

The study examined experience of domestic violence, which should be contrasted with perpetration. Domestic violence was defined by a 'yes' response to a number of questions that described domestic violence, including physical, sexual and emotional violence by the participants' current and former partner. The questionnaire applied the Home Office case definition of domestic violence, being 'any violence between current and former partners in an intimate relationship, wherever the violence occurs. The violence may include physical, sexual, emotional and financial abuse' (www.justice.gov.uk/about/domesticviolence.htm). Women were also asked about the acceptability of being questioned by healthcare workers about domestic violence by a series of questions, for example: 'In general, would you mind if your psychiatrist asked you whether you were being threatened, hit or hurt by your partner or a previous partner?' The selfadministered questionnaire was piloted and modified, and thereafter completed in the presence of the researchers.

Data were entered, checked for accuracy and analysed using SPSS version 14 for Windows. Univariate analyses were conducted using the $\chi^{2}$-test for frequencies or Fisher's exact test where appropriate. Logistic regression analysis was used to identify risk factors relating to domestic 
violence. Sample size calculations showed that 64 participants were needed to have $90 \%$ power to show a $15 \%$ difference in a range of demographic variables and to be significant at the 0.05 level between women who had experienced physical violence.

Participants' ethnic groups were based on categories defined by the Office for National Statistics. ${ }^{8}$ We restricted the study to female patients because of the high prevalence of domestic violence among women in the general population and the gender of the researchers, as suggested by the WHO ethics guidelines for research into domestic violence. ${ }^{9}$

The questions looked at: demographic information including past or present relationship; psychiatric diagnosis; self-harm; history of childhood abuse; different categories of domestic violence; women's attitudes to being questioned by psychiatrist or key worker about abuse; and any previous disclosure. Participants were invited to record their comments about the study. Data were collected on diagnoses and any disclosure of domestic violence documented in the case notes.

\section{Results}

Overall, 71 women agreed to participate in the study and to examination of their case notes; 1 did not complete the questionnaire as she had never been in a relationship and 18 women refused $(n=71 / 89,80 \%$ participation rate). Demographic differences between participants and nonparticipants were not analysed, as non-participants did not consent to such an analysis.

The mean age of participants was 50 years (s.d.=13.9, median 49.8). Most women had been in a close relationship in the past $(n=53,61 \%)$ but only 27 had a partner at the time of the study (38\%). The majority also had children $(n=47,66 \%)$ and were not working $(n=50,70 \%)$, with 31 receiving sickness benefit (44\%) and 21 employed (30\%).

Forty-four $(62 \%)$ women were born in the UK. The majority $(n=51,72 \%)$ were White and 15 were Black (Caribbean and African, 21\%), which is higher than the average for the population of Wandsworth (8\%). The participants had a lower home ownership rate than the local population $(35 \%$ v. $52 \%)$, with a greater part living in council or housing association accommodation $(n=40,56 \%)$.

Diagnoses were obtained from psychiatric case notes. The primary diagnosis was schizophrenia/delusional disorder in 16 participants $(22 \%)$, bipolar schizoaffective disorder in 16 (22\%), depressive disorder in $14(20 \%)$, anxiety disorder in 10 (14\%), personality disorder in 8 (11\%), eating disorder in $2(3 \%)$, alcohol dependence in $3(4 \%)$ and drug dependence in $2(3 \%)$. Overall, 46 women $(65 \%)$ had been admitted to a psychiatric hospital in the past and 20 women (28\%) had been detained under the Mental Health Act. Of 62 women on medication (87\%), 33 were on antipsychotics or mood stabilisers or both (53\%). Over half our sample reported self-harm, with 23 having ever taken an overdose (33\%) and 29 having cut, burnt or hurt themselves in other ways (41\%).

\section{Prevalence}

Experiences of specific categories of domestic violence are shown in Table 1. We make reference predominantly to lifetime data as only a small number of women had a current partner in the past 12 months. However, Table 1 includes some data on violence in the past 12 months,

\begin{tabular}{|c|c|c|}
\hline Type of violence & $n(\%)$ & $95 \% \mathrm{Cl}$ \\
\hline Do you think you have ever experienced domestic violence? & $37(52)$ & $40.6-64.9$ \\
\hline Physical violence by partner & $42(60)$ & $47.6-71.5$ \\
\hline Grabbed or shoved you & $31(43)$ & $32.4-56.7$ \\
\hline Punched you on body/arms/legs & $25(36)$ & $24.6-48.1$ \\
\hline Punched you in the face & $19(27)$ & $17.2-39.1$ \\
\hline Forced you to have sex & $24(33)$ & $23-46.6$ \\
\hline Physically violent to you in other way & $20(27)$ & $18.4-40.6$ \\
\hline Kicked you on the floor & $16(24)$ & $13.7-34.4$ \\
\hline Choked or held hand over your mouth & $19(26)$ & $17.2-39.1$ \\
\hline Used weapon or object to hurt you & $13(19)$ & $10.3-29.7$ \\
\hline Tried to strangle, burn or drown you & $10(13)$ & $24.7-70.7$ \\
\hline Threatening behaviour by partner & $38(54)$ & $41.9-66.3$ \\
\hline Punched, kicked or threw things & $31(44)$ & $32.4-56.7$ \\
\hline Threatened you with fist, hand or foot & $29(40)$ & $29.8-53.8$ \\
\hline Threatened you with object or weapon & $20(29)$ & $18.4-40.6$ \\
\hline Threatened to kill you & $17(24)$ & $14.8-36$ \\
\hline Controlling behaviour by partner & $57(81)$ & $70.3-89.7$ \\
\hline Shouted, screamed or swore at you & $48(69)$ & $56.4-79.1$ \\
\hline Criticised you & $52(74)$ & $62.4-84$ \\
\hline Checked up on your movements & $31(44)$ & $33.7-58.1$ \\
\hline Restricted your social life & $37(53)$ & $40.6-64.9$ \\
\hline Tried to control you in any other way not physical violence & $34(49)$ & $37.8-62.2$ \\
\hline Kept you short of money & $30(43)$ & $29.8-53.8$ \\
\hline Locked you in the house & $10(15)$ & $24.7-70.7$ \\
\hline Physical violence in the past 12 months & $11(15)$ & $26.4-81.1$ \\
\hline Have you ever felt afraid of your partner? & $41(59)$ & $46.2-70.2$ \\
\hline Domestic violence during pregnancy & $14(27)$ & $11.4-31.3$ \\
\hline True rate of recording of domestic violence in case notes & $18(26)$ & $15.8-37.1$ \\
\hline
\end{tabular}

a. Seventy-one women agreed to participate but one woman did not complete the questionnaire as she had never been in a relationship 
within the limits of a small data-set. In total, $60 \%$ of women $(n=42)$ had experienced physical violence from their current or previous partner, $81 \%(n=57 / 70)$ had experienced some form of controlling behaviour and $54 \%(n=38)$ had been threatened by their current or previous partner. A smaller proportion $(52 \%, n=37)$ responded positively to the question 'Do you think you have ever experienced domestic violence?' Domestic violence was not uncommon during pregnancy and was reported by 14 of the study population $(n=70,27 \%)$, of whom a quarter ascribed worsening violence to the pregnancy and $12 \%(n=3 / 25)$ attributed miscarriage to the violence.

\section{Disclosure and recording in the clinical notes}

Of 65 women who had experienced domestic violence, $40 \%$ $(n=26)$ reported physical injuries as a direct result but only half sought medical attention. Regarding disclosure, of 39 women who answered the question, $51 \%$ reported disclosure of domestic violence to the psychiatrist and $29 \%$ to their general practitioner (GP). The majority of participants had never been questioned by a psychiatrist or other health professional about experiences of domestic violence, with only $22 \% \quad(n=16)$ having received questions from a psychiatrist or key worker about threatened or actual physical violence and $13 \%(n=9)$ about sexual violence by their partner. Addressing attitudes and acceptability of such questioning, the vast majority $(n=58,82 \%)$ regarded questions from a psychiatrist or key worker as entirely acceptable, with $10 \%$ objecting and the same proportion 'unsure'. There were no demographic or clinical differences between those objecting and those not, which may represent a type II error. Gender of psychiatrist was considered relevant by most participants, with $53 \% \quad(n=38)$ expressing a preference for a woman and $39 \%(n=28)$ unconcerned by gender. Domestic violence as a precipitant of hospital admission was also examined by medical note review, but no record of this was found. In only 18 of the women's case notes $(25 \%)$ was there any record of domestic violence.

\section{Variables associated with domestic violence}

There was no statistically significant association between domestic violence and age, employment, ethnic group or being born in the UK (online Table DS1). There were significant associations with having experienced actual physical violence (as opposed to threatening or controlling behaviour) ( $n=42,60 \%)$, having taken an overdose $(n=22$, $54 \%)$ and having children $(n=32,78 \%)$. Cohabiting with a partner was protective $(n=7,19 \%)$. Admission to psychiatric hospital or being detained under the Mental Health Act did not put women at more risk of domestic violence.

\section{Sexual violence}

About half of those women who had suffered violence $(n=23,55 \%)$ had also experienced sexual violence by their partner. In addition, 18 women (25\%) had experienced sexual abuse before the age of 16 and $25(35 \%)$ since the age of 16 years (online Table DS1).

\section{Interrelationships between variables}

Logistic regression analyses were used to identify demographic variables that were significantly related to domestic violence. For the purpose of this analysis, we included any woman who had ever experienced any type of physical violence. We defined current domestic violence as physical violence experienced during the past 12 months. We constructed a logistic regression model to assess the influence of all those variables found to be significant in influencing outcome (physical violence): presence of children, a history of overdose, and experience of sexual abuse before and after the age of 16 years. Variables were entered in a forward stepwise manner; they were considered as significant at the $5 \%$ level. The presence of children, a history of overdose, and experience of sexual abuse after the age of 16 years at presentation were the only variables found to have a significant independent effect on experiencing physical violence (Table 2).

\section{Discussion}

This is the first prevalence study of domestic violence in a non-emergency psychiatric setting in the UK, and shows that $60 \%$ of female patients had suffered physical domestic violence at some point in their lives. A very high proportion suffered from actual physical injuries but only half sought medical attention. Of those who were victims, $27 \%$ had experienced domestic violence during pregnancy. These figures are higher than in other healthcare settings or in the general population, particularly for physical violence., ${ }^{2,3}$ Chronic emotional abuse may be just as relevant to the pathogenesis of mental illness and disorder, and rates are also very high in this study. One of the authors (J.F.M.) is currently examining the role of dissociation as a mediating factor between domestic violence and eating disorders, and emotional rather than physical abuse appears pertinent.

\section{Disclosure}

Despite high prevalence, domestic violence was only recorded in clinical notes in $26 \%$ of cases, although the majority of women found questions regarding violence acceptable.

Our study replicates findings in primary care ${ }^{2}$ in terms of acceptability of routine enquiry for domestic violence: most women were in favour, with a minority objecting. Health professionals show reticence towards enquiring about violence; they do not feel trained or equipped for effective interventions. ${ }^{10}$ Concerns have been expressed that routine enquiry may also endanger the women, such that it has not been recommended except in high-risk groups such as pregnant women.

This study suggests that women with mental disorders should also be included in high-risk groups, and psychiatric health workers should be equipped to enquire about violence. In a systematic review, Ramsay et $a l^{10}$ suggest that routine enquiry without evidence of effective interventions should not be recommended. On the contrary, this study supports routine enquiry in psychiatric services. In our sample, $69 \%$ of the women had been admitted to a psychiatric hospital

\begin{tabular}{lcc|}
\hline Table 2 & \multicolumn{3}{l}{$\begin{array}{l}\text { Logistic regression analysis of characteristics } \\
\text { associated with domestic violence }\end{array}$} \\
& $P$ & Odds ratio $(95 \% \mathrm{Cl})$ \\
\hline Children & 0.05 & $3.34(0.97-11.49)$ \\
\hline Overdose & 0.02 & $4.32(1.24-14.93)$ \\
\hline $\begin{array}{l}\text { Unwanted sexual experience } \\
\text { since age 16 years }\end{array}$ & 0.002 & $6.76(2.00-22.73)$ \\
\hline
\end{tabular}


and $30 \%$ had been detained under the Mental Health Act, indicating the severity of their illnesses and probably their increased vulnerability to become victims of violence. These findings alone should justify, from a human rights perspective and on ethical grounds, enquiries about domestic violence. In addition, the Royal College of Psychiatrists' policy statement on domestic violence ${ }^{11}$ recommends that enquiry on domestic violence should be part of the routine clinical assessment. The Victims of Violence and Abuse Prevention Programme has established a pilot to introduce routine clinical enquiry about violence and abuse as a component of the care programme approach. ${ }^{12}$

\section{Risk factors}

Univariate analysis showed that those abused by their partner were more likely to have attempted suicide by taking overdoses, to use street drugs, to have had children and to have suffered sexual abuse before and since the age of 16 years. The significant association between physical abuse and a history of overdose confirms data from other studies of high levels of depression as a consequence of domestic violence. ${ }^{13,14}$ The particular risk to mothers highlights the need to consider issues of safeguarding children in such cases. The literature has identified that women under 40 years of age who are single, separated or divorced are at higher risk. ${ }^{15}$ We did not find any association between violence and age. This might be due to the fact that the study population was older and that only a small number were in a relationship $(n=27,38 \%)$.

The history of sexual abuse after the age of 16 replicates the findings of the study by Coid et al. ${ }^{14}$ We cannot assert any causal link in view of study design, small sample and lifetime prevalence as outcome measure. However, a disproportionately high number of women suffering sexual abuse is worthy of note: 18 before the age of 16 and 25 after.

A smaller proportion $(52 \%, n=37)$ responded positively to the question 'Do you think you have ever experienced domestic violence?', which replicates findings in a primary care setting. ${ }^{2}$ Women who have suffered violence at times do not recognise it as such, or may think they 'deserved' it. ${ }^{2}$

\section{Physical injuries}

Physical injuries were reported by $42 \%$ of the women, but only half sought medical attention. These are very high rates, explicable in a psychiatric population by the impact of physical injuries on mental health, and by the vulnerability of women with severe mental disorders. However, because of the small numbers we cannot attribute causality.

\section{Study limitations}

We cannot be confident that our results generalise to other psychiatric populations, although the findings replicate those of other studies and we achieved a high response rate for work in this field. We have not examined prevalence among male psychiatric patients, and comparable studies are needed. The study demonstrates associations, but it was not constructed to test hypotheses concerning causal relations. The study was not controlled and it is possible that higher rates of violence reflect the London population from which the sample was drawn.
This study demonstrates that domestic violence in female psychiatric patients is much more common than in other patient groups. Most female psychiatric patients have experienced domestic violence but almost half have not disclosed it. Disclosure to a psychiatrist is rare, but acceptable to most patients. We suggest that routine enquiry about domestic violence should be considered in women who experience serious mental illness, and women should receive help with domestic violence as part of their care. The nature of that help would be determined by circumstances, but should include social and legal advice, as well as emotional support focused on safety planning.

\section{About the authors}

John F. Morgan, Senior Lecturer in Psychiatry, Leeds, and St George's University, London, Gabriella Zolese, Consultant Psychiatrist, South West London and St George's Mental Health NHS Trust, Jane McNulty, Consultant Psychiatrist, Sussex Partnership NHS Trust, and Sharon Gebhardt, Consultant Psychiatrist, Surrey and Borders Partnership NHS Trust.

\section{References}

1 Garcia-Moreno C, Jansen HAFM, Ellsberg M, Watts CH. Prevalence of intimate partner violence: findings from the WHO multi-country study on women's health and domestic violence. Lancet 2006; 368: 1260-9.

2 Richardson J, Coid J, Petruckevitch A, Chung W, Moorey S, Feder G. Identifying domestic violence: cross sectional study in primary care. BMJ 2002; 324: 274 .

3 Sethi D, Watts S, Zwi A, Watson J, McCarthy C. Experience of domestic violence by women attending an inner city accident and emergency department. Emerg Med J 2004; 21: 180-4.

4 Golding JM. Intimate partner violence as a risk factor for mental disorders: a meta-analysis. J Fam Violence 1999; 14: 99-132.

5 Cascardi M, Mueser KT, De Girolamo J, Murrin M. Physical aggression against psychiatric inpatients by family members and partners. Psychiatr Serv 1996; 47: 531-3.

6 Miller LJ, Finnerty M. Sexuality, pregnancy, and childrearing among women with schizophrenia-spectrum disorders. Psychiatr Serv 1996; 47: 502-6.

7 Tham SW, Ford TJ, Wilkinson DG. A survey of domestic violence and other forms of abuse. J Ment Health 1995; 4: 317-21.

8 Dobbs J, Green H, Zealey L (eds). Focus on Ethnicity and Religion: 5. Palgrave Macmillan.

9 World Health Organization. Putting Women First: Ethical and Safety Recommendations for Research on Domestic Violence against Women. WHO, 2001.

10 Ramsay J, Richardson J, Carter YH, Davidson LL, Feder G. Should health professionals screen women for domestic violence? Systematic review. BMJ 2002; 325: 314-25.

11 Royal College of Psychiatrists. Domestic Violence (Council Report CR102). Royal College of Psychiatrists, 2002.

12 Itzin C, Bailey S, Bentovim A. The effects of domestic violence and sexual abuse on mental health. Psychiatr Bull 2008; 32: 448-50.

13 Straus MA, Gelles RJ. Physical Violence in American Families: Risk Factors and Adaptations to Violence in 8,145 Families. Transaction Publishers, 1990.

14 Coid J, Petruckevitch A, Feder G, Chung W, Richardson J, Moorley S Relation between childhood sexual and physical abuse and risk of revictimisation in women: a cross-sectional survey. Lancet 2001; 35: 450-4.

15 Dearwater SR, Coben JH, Campbell JC, Nah G, Glass N, McLaughlin E, et al. Prevalence of intimate partner abuse in women treated at community hospital emergency departments. JAMA 1998; 280: 433-8. 